\title{
An Implicit Plan Still Overrides an Explicit Strategy During Visuomotor Adaptation Following Repetitive Transcranial Magnetic Stimulation of the Cerebellum
}

\author{
Sarah H. E. M. Voets ${ }^{1}$, Muriel T. N. Panouilleres ${ }^{2}$ and Ned Jenkinson ${ }^{1,3 \star ~(D) ~}$ \\ ${ }^{1}$ School of Sport, Exercise, and Rehabilitation Sciences, University of Birmingham, Birmingham B15 2TT, UK, ${ }^{2}$ Nuffield \\ Department of Clinical Neurosciences, Level 6, West Wing, John Radcliffe Hospital, Oxford OX3 9DU, UK, and ${ }^{3}$ Centre for \\ Human Brain Health, University of Birmingham, Birmingham B15 2TT, UK \\ Corresponding author. E-mail: n.jenkinson@bham.ac.uk
}

(Received 15 January 2020; Revised 12 March 2020; Accepted 12 March 2020).

\begin{abstract}
Motor adaptation is a process by which the brain gradually reduces error induced by a predictable change in the environment, e.g., pointing while wearing prism glasses. It is thought to occur via largely implicit processes, though explicit strategies are also thought to contribute. Research suggests a role of the cerebellum in the implicit aspects of motor adaptation. Using non-invasive brain stimulation, we sought to investigate the involvement of the cerebellum in implicit motor adaptation in healthy participants. Inhibition of the cerebellum was attained through repetitive transcranial magnetic stimulation (rTMS), after which participants performed a visuomotor-rotation task while using an explicit strategy. Adaptation and aftereffects of the TMS group showed no difference in behaviour compared to a Sham stimulation group, therefore this study did not provide any further evidence of a specific role of the cerebellum in implicit motor adaptation. However, our behavioral findings replicate those in the seminal study by Mazzoni and Krakauer (2006).
\end{abstract}

Keywords: cerebellum; implicit; explicit; motor adaptation; TMS; visuomotor rotation

\section{Introduction}

The exact contributions of explicit and implicit processes to motor adaptation are unknown. While the cerebellum is thought to have a major role in the implicit processes, it is thought that other brain areas contribute more explicit processes (Taylor et al., 2014). In a seminal paper, Mazzoni and Krakauer (2006) demonstrated that implicit adaptation overrides explicit strategy in a visuomotor-rotation task. Though the explicit strategy led to an instant correction of the error, participants started to drift away from the target ending close to their aiming location, i.e. implicit adaptation started to occur around the aiming location instead of the target and overrode the strategy - this rather counterintuitive behavioural finding has not been directly replicated. Interestingly, patients with cerebellar degeneration can better use strategy for adaptation compared to controls where performance deteriorates (Taylor et al., 2010), suggesting a primary role of the cerebellum in implicit, but not explicit motor adaptation.

\footnotetext{
(C) The Author(s) 2020. This is an Open Access article, distributed under the terms of the Creative Commons Attribution licence (http:// creativecommons.org/licenses/by/4.0/), which permits unrestricted re-use, distribution, and reproduction in any medium, provided the original work is properly cited.
} 


\section{Objective}

To investigate the cerebellum's role in implicit motor adaptation by inhibiting its function with $1 \mathrm{~Hz}$ rTMS before, participants performed a $45^{\circ}$ counter-clockwise visuomotor-rotation task using an explicit strategy. rTMS has previously been used successfully to inhibit the cerebellum and produce behavioural changes (see Théoret et al., 2001; Miall \& Christensen, 2004; Jenkinson \& Chris Miall, 2010). If the cerebellum is responsible for the implicit motor adaptation that has been shown to drive the participant away from the target (Mazzoni \& Krakauer, 2006) after initially correcting for the rotation using strategy, inhibition of the cerebellum should reduce the speed or size of the drift towards the aiming target and result in sustained explicit compensation of the rotation.

\section{Methods}

Stimulation ( $\mathrm{N}=12: 7$ women, age $27 \pm 2$, range 18-36) was applied using a double-cone coil at $55 \%$ of maximum-stimulator output (MSO) (MagStim Super Rapid) (Jenkinson \& Chris Miall, 2010) over the right cerebellar hemisphere, $1 \mathrm{~cm}$ below and $3 \mathrm{~cm}$ lateral from the inion. Sham stimulation $(\mathrm{N}=10$ : 8 women, age $24 \pm 2$, range 20-39) was performed with a flat figure-of-eight coil at $35 \% \mathrm{MSO}, 4 \mathrm{~cm}$ below and $6 \mathrm{~cm}$ lateral from the inion, which induced muscle twitches around the neck that were similar to the active cerebellar stimulation without targeting the cerebellum (Hardwick et al., 2014). All participants were blind to the effectiveness of stimulation and received 600 pulses of $1 \mathrm{~Hz}$ TMS before they performed a visuomotor-rotation task with a joystick similar to Mazzoni and Krakauer (2006). See Figure 1 for details.

\section{Results}

No group differences in directional error (DE) were found for baseline 1 (average last 2 trials); $\mathrm{t}(20)=.944, \mathrm{p}=.357$ and baseline $2 ; \mathrm{t}(20)=.582, \mathrm{p}=.567$, confirming similar behaviour before adaptation. $\mathrm{DE}$ during the initial two trials of rotation did not differ between the groups; $\mathrm{t}(15.7)=.161, \mathrm{p}=.874$. The use of strategy initially helped overcome the rotation, but implicit adaptation overrode this explicit strategy

a.

\begin{tabular}{|c|c|c|c|c|c|c|}
\hline $\begin{array}{c}\text { Baseline } 1 \\
40 \text { trials }\end{array}$ & POAds & $\begin{array}{c}\text { Baseline } 2 \\
24 \text { trials }\end{array}$ & $\begin{array}{c}\text { Rotation } \\
2 \text { trials }\end{array}$ & מnstracton & $\begin{array}{c}\text { Rotation } \\
+ \text { Strategy } \\
72 \text { trials }\end{array}$ & $\begin{array}{l}\text { Washout } \\
72 \text { trials }\end{array}$ \\
\hline
\end{tabular}

b.

\section{Baseline}

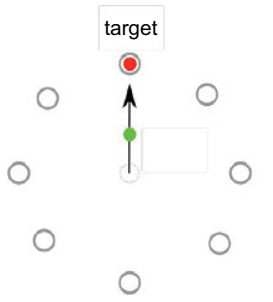

Rotation

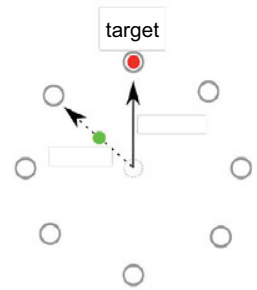

Strategy

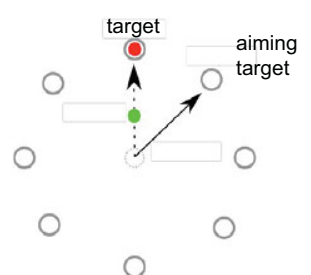

Washout

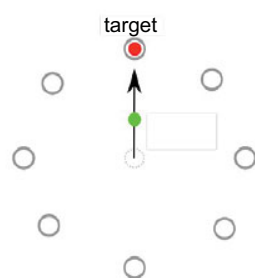

Figure 1. Experimental paradigm (a) and visuomotor-rotation task (b). Participants performed 40 trials without rotation (Baseline 1), before receiving 10 minutes of rTMS, followed by another 24 trials without rotation (Baseline $2 ; \sim 2.5$ mins). They were then exposed to two trials where a $45^{\circ}$ counter-clockwise rotation was imposed on the cursor output before being instructed to use strategy. The strategy entailed to aim for the clockwise neighbouring target next to where the red cursor would appear. After the instruction participants performed another 72 trials of rotation (Rotation + Strategy; $\sim 7$ mins) and then 72 trials where the rotation was removed and no strategy had to be used (Washout; 7 mins). 


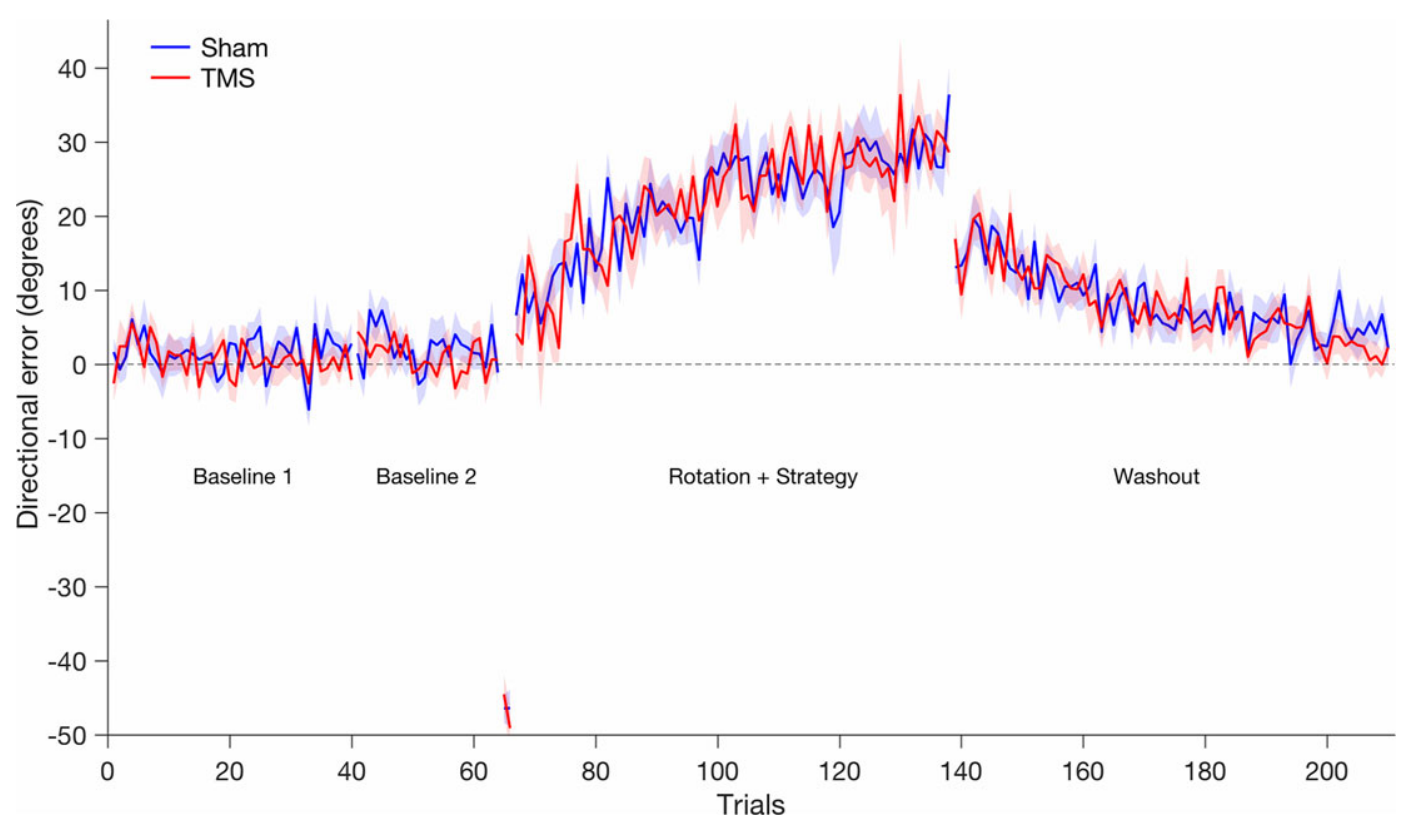

Figure 2. Average directional error across all trials during the five phases (Baseline 1, Baseline 2, 2 trials Rotation, Rotation + Strategy, and Washout) for both the Sham (blue) and TMS (red) group.

around the aiming target in both groups (see Figure 2). TMS did not have an effect on DE during early; $\mathrm{t}(20)=.920, \mathrm{p}=.368$ and late adaptation; $\mathrm{t}(20)=.093, \mathrm{p}=.927$, nor during early; $\mathrm{t}(20)=.150, \mathrm{p}=.882$ or late washout; $\mathrm{t}(20)=1.761, \mathrm{p}=.093$ (first and last 8 trials).

\section{Discussion}

We found that rTMS of the cerebellum had no effect on implicit adaptation; in both groups implicit adaptation overrode explicit strategy. The stimulation site used is thought to correspond to cerebellar lobule VIII (Manni \& Petrosini, 2004), and has been used for cerebellar studies investigating hand use (Théoret et al., 2001). Though hand representations are mainly thought to be in lobule VIII, there are representations in lobule V (Manni \& Petrosini, 2004; Wiestler et al., 2011). However, this may be an underestimate of the distribution (see Mottolese et al., 2012) which may have prevented us from inhibiting all the representations. Regardless of the negative finding, this interesting behavioural paradigm demonstrating the strength of implicit motor adaptation appears robust and replicable.

\section{Conclusions}

Contrary to our hypothesis, $1 \mathrm{~Hz}$ TMS of the cerebellum did not result in a reduced implicit drift. Therefore, we are left with two likely conclusions:

1. The cerebellum was inhibited, but inhibition of the cerebellum had no influence on implicit motor adaption.

2. The stimulation failed to inhibit the cerebellum, or the area of the cerebellum was not the area (or areas) of the cerebellum controlling adaptation of the hand.

Our results represent an important replication of a rather counterintuitive behavioural finding, and emphasizes the strength of the implicit processes that take place during motor adaptation (wherever these processes takes place) and the lack of influence that explicit strategy has upon these processes. 
Author contribution. NJ \& SV conceived and designed the study. SV, MP \& NJ conducted data gathering. SV performed statistical analyses. SV \& NJ wrote the article.

Funding information. This work was supported by funding from both Parkinson's UK (H-1402 \& G-1108) and the Medical Research Council (MR//J004588/1).

Conflict of interest. The authors NJ, MP \& SV declare none.

Ethics statement. The authors assert that all procedures contributing to this work comply with the ethical standards of the relevant national and institutional committees on human experimentation and with the Helsinki Declaration of 1975, as revised in 2008

Data Availability Statement. The data that support the findings of this study are openly available upon request to the corresponding author.

\section{REFERENCES}

Hardwick, R. M., Lesage, E., \& Chris Miall, R. (2014). Cerebellar transcranial magnetic stimulation: The role of coil geometry and tissue depth. Brain Stimulation, 7, 643-649. https://doi.org/10.1016/j.brs.2014.04.009.

Jenkinson, N., \& Chris Miall, R. (2010). Disruption of saccadic adaptation with repetitive transcranial magnetic stimulation of the posterior cerebellum in humans. Cerebellum, 9, 548-555. https://doi.org/10.1007/s12311-010-0193-6.

Manni, E., \& Petrosini, L. (2004). A century of cerebellar somatotopy: A debated representation. Nature Reviews Neuroscience, $5,1-9$.

Mazzoni, P., \& Krakauer, J. W. (2006). An implicit plan overrides an explicit strategy during visuomotor adaptation. The Journal of Neuroscience, 26, 3642-3645. https://doi.org/10.1523/JNEUROSCI.5317-05.2006.

Miall, R. C., \& Christensen, L. O. D. (2004). The effect of rTMS over the cerebellum in normal human volunteers on peg-board movement performance. Neuroscience Letters, 371, 185-189. https://doi.org/10.1016/j.neulet.2004.08.067.

Mottolese, C., Richard, N., Harquel, S., Szathmari, A., Sirigu, A., \& Desmurget, M. (2012). Mapping Motor Representations in the Human Cerebellum. Brain, 136, 330-42. https://doi.org/10.1093/brain/aws186

Taylor, J. A., Klemfuss, N. M., \& Ivry, R. B. (2010). An explicit strategy prevails when the cerebellum fails to compute movement errors. Cerebellum, 9, 580-586. https://doi.org/10.1007/s12311-010-0201-x.

Taylor, J. A., Krakauer, J. W., \& Ivry, R. B. (2014). Explicit and implicit contributions to learning in a sensorimotor adaptation task. The Journal of Neuroscience, 34, 3023-3032. https://doi.org/10.1523/JNEUROSCI.3619-13.2014.

Théoret, H., Haque, J., \& Pascual-Leone, A. (2001). Increased variability of paced finger tapping accuracy following repetitive magnetic stimulation of the cerebellum in humans. Neuroscience Letters, 306, 304-307.

Wiestler, T., McGonigle, D. J., \& Diedrichsen, J. (2011). Integration of sensory and motor representations of single fingers in the human cerebellum. Journal of Neurophysiology, 105, 3042-3053. https://doi.org/10.1152/jn.00106.2011.

Cite this article: H. E. M. Voets S, Panouilleres MTN, Jenkinson N (2020). An Implicit Plan Still Overrides an Explicit Strategy During Visuomotor Adaptation Following Repetitive Transcranial Magnetic Stimulation of the Cerebellum Experimental Results, 1, e16, 1-8. https://doi.org/10.1017/exp.2020.16 


\section{Peer Reviews}

\section{Reviewing editor: Dr. Michael Nevels}

University of St Andrews, Biomolecular Sciences Building, Fife, United Kingdom of Great Britain and Northern Ireland, KY16 9ST

This article has been accepted because it is deemed to be scientifically sound, has the correct controls, has appropriate methodology and is statistically valid, and met required revisions.

doi:10.1017/exp.2020.16.pr1

Review 1: An Implicit Plan Still Overrides an Explicit Strategy During Visuomotor Adaptation Following Repetitive Transcranial Magnetic Stimulation of the Cerebellum

\section{Reviewer: Pietro Mazzoni i}

Deakin University 2104 Psychology Burwood Victoria Australia 3125

Date of review: 29 February 2020

Published online:

(C) The Author(s) 2020. This is an Open Access article, distributed under the terms of the Creative Commons Attribution licence http://creativecommons.org/licenses/by/4.0/), which permits unrestricted re-use, distribution, and reproduction in any medium, provided the original work is properly cited.

Conflict of interest statement. Reviewer declares none.

Comments to the Author: The negative result is interesting but requires commenting on whether the protocol was adequate to achieve cerebellar inhibition. I suggest pointing out that the protocol appropriately reproduced elements of two previous studies that achieved motor task disruption through cerebellar rTMS (Theoret et al. 2001, and Jenkinson and Miall 2010) but pulse intensity (35\% of max. stimulator output) was lower than in Jenkinson and Miall 2010 (who used 45\%, 55\% of MSO and the same coil type). It could be argued that 35\% could have been adequate because Jenkinson \& Miall targeted the oculomotor vermis, and if this region is deeper than the region targeted here then $35 \%$ could have been adequate. The present study was well designed, with attention to details of rTMS protocol that make the study of interest even if the result is negative. Indeed, the authors should point out more explicitly that their protocol reproduced the crucial features of protocols that previously showed inhibitory effect on cerebellar tissue. Then they should acknowledge that the pulse intensity they chose may have been inadequate to inhibit cerebellar circuits responsible for hand visuomotor adaptation, and that this may be an alternative explanation for the negative result.The language has minor errors including some syntax that makes a few sentences unclear. I suggest that the authors have the manuscript edited to smooth out these minor unclear spots. Examples: lines 31, 33, 37, 46, 77.

\section{Score Card}

Presentation

Does the paper cite relevant and related articles appropriately? (30\%) 
Does the abstract correctly embody the content of the article? (25\%)

Does the introduction give appropriate context? (25\%)

Is the objective of the experiment clearly defined? (25\%)

Analysis

Does the discussion adequately interpret the results presented? (40\%)

Is the conclusion consistent with the results and discussion? (40\%)

Are the limitations of the experiment as well as the contributions of the experiment clearly outlined? (20\%) 


\section{Review 2: An Implicit Plan Still Overrides an Explicit Strategy During Visuomotor Adaptation Following Repetitive Transcranial Magnetic Stimulation of the Cerebellum}

Reviewer: Dr. Lara Fernandez

Deakin University, Psychology, Burwood, Victoria, Australia, 3125

Date of review: 02 April 2020

Published online:

(c) The Author(s) 2020. This is an Open Access article, distributed under the terms of the Creative Commons Attribution licence http://creativecommons.org/licenses/by/4.0/), which permits unrestricted re-use, distribution, and reproduction in any medium, provided the original work is properly cited.

Conflict of interest statement. Reviewer declares none.

Comments to the Author: The following study investigated the effects of cerebellar inhibition via rTMS on implicit adaptation in an upper-limb visuomotor rotation task. This was generally a well designed experiment, following protocols for cerebellar stimulation and visuomotor adaptation assessment as defined in previous literature. However, one design aspect that should have been considered is the total time taken post-rTMS for the task to be completed. The effects of repetitive TMS on cerebellar tissue, including duration-related factors, are currently not well understood. Thus, if the post-rTMS trial time was particularly lengthy, it may be possible that the effects of the stimulation had diminished by the time the participant performed rotation+strategy trials. Therefore, the time taken to complete each trial block should be added to Figure 1. An assessment of cerebellar brain inhibition (CBI) should ideally have been performed at several relevant time-points following rTMS (see Popa, Russo, Meunier (2010)). This would give some indication of the extent to which the stimulation was having on the anterior cerebellum (primarily motor region). Notably, in Jenkinson and Miall (2010), the intensity of 55\% MSO was believed to be targeting posterior regions for the disruption of saccade-related adaptation. Hence, this intensity may not be appropriate for limb-related adaptation tasks (where higher intensities may be required). CBI should also be assessed pre- and post-adaptation to see if the task was likely to be activating the cerebellum. These additional assessments may be performed and added to the existing study as 'pilot data', though, ideally the same participants would be used.

\section{Score Card}

Presentation

3.3

Is the article written in clear and proper English? (30\%)

Is the data presented in the most useful manner? (40\%)

Does the paper cite relevant and related articles appropriately? (30\%)

\section{Context}

Does the abstract correctly embody the content of the article? (25\%)

Does the introduction give appropriate context? (25\%)

Is the objective of the experiment clearly defined? (25\%) 
Are the limitations of the experiment as well as the contributions of the experiment clearly outlined? (20\%) 